\title{
LA POBLACIÓN DESPLAZADA EN CARTAGENA DE INDIAS: ALCANCES Y LIMITACIONES DE LA POLÍTICA PÚBLICA
}

DISPLACED PEOPLE IN CARTAGENA DE INDIAS: SCOPE AND LIMITATIONS

OF PUBLIC POLICY

Por: Amaranto Daniels Puello", Raúl Román Romero"“ y Jennifer Jiménez Garć́a"”". Artículo Recibido: Mayo 30 de 2010. Artículo Aprobado: Junio 30 de 2010. Páginas: 40-57

*AMARANTO DANIELS

PUELLO

Economista (Universidad de Cartagena), Magíster en Estudios Politico-Económicos y Docente de la Universidad de Cartagena (Colombia), invesCartagena (Colombia), investigador del Instituto Internacional de Estudios del Caribe. E-mail: amarantodaniels@ hotmail.com

**RAÚl ROMÁN ROMERO Historiador (Universidad de Cartagena). Candidato a Doctor en Historia de América Latina. Docente y coordinador de la Maestría en Estudios del Caribe de la Universidad Nacional de Colombia, sede Caribe (Colombia). E-mail: raulromanr@yahoo.com

***JENNIFER JIMÉNEZ GARCÍA

Psicóloga. Candidata a Magíster en Desarrollo Social. Universidad del Norte (Cotombia).

Artículo tipo 1 de investigación científica y tecnológica según clasificación Colciencias.

\section{RESUMEN}

Este artículo analiza los alcances y limitaciones que ha tenido la política pública sobre la población desplazada en la ciudad de Cartagena, así mismo evalúa las condiciones de vulnerabilidad que enfrenta la población desplazada frente a las precariedades que tiene el gobierno distrital para hacer frente a la grave situación de exclusión rechazo e inserción social en que se ve envuelta esta población.

Palabras clave: Desplazamiento forzado, política pública, conflicto armado, Cartagena, pobreza.

\section{ABSTRACT}

This article analyzes the scope and limitations that the public policy has taken on the displaced population in the city of Cartagena, at the same way evaluates the vulnerable conditions that faces the displaced people against the precariousness that the district government addresses to the serious refusal situation of exclusion and social integration in which this population is involved.

Keywords: Forced displacement, public policy, armed conflict, Cartagena, poverty.

\section{INTRODUCCIÓN}

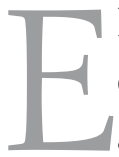
l conflicto armado, la violencia y la reconciliación entre los colombianos, constituye uno de los problemas centrales que afronta el país en los actuales momentos. Es importante precisar que estos aspectos no son los únicos que demandan una atención inmediata del Estado, ya que a los problemas referenciados, hay que sumar, la frágil unidad nacional, la desintegración social, el incremento de la pobreza y la exclusión. El escenario descrito se ha venido empeorando, entre otros factores, por la manifestación del desplazamiento forzado, el cual ante su crecimiento continuo en los últimos veinte años se ha convertido en uno de los fenómenos que representa una amenaza para la integración social y en consecuencia para la unidad de la 


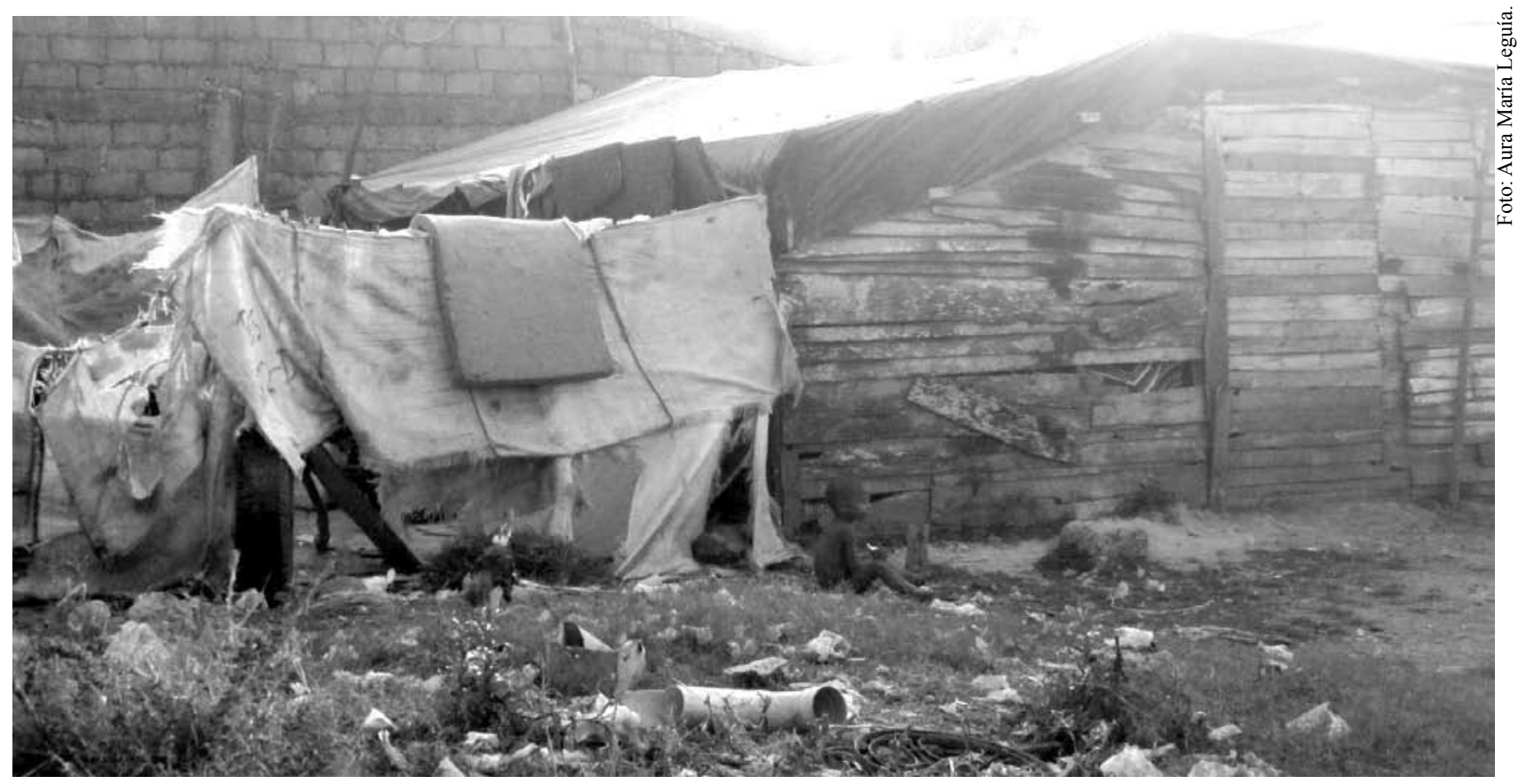

Lindero. Cartagena 2011.

nación; sobre todo si el Estado no asume de manera responsable una política pública integral para hacer frente a la tragedia humanitaria que padecen miles de familias desterradas y en constante peregrinación por el territorio nacional.

Aunque Colombia no es el único país que enfrenta esta situación, ya que el desplazamiento se convirtió en uno de los fenómenos más alarmantes del mundo actual, (según ACNUR en su informe del año 2008 en el mundo se estiman 42 millones de desplazados) es innegable que el tema en este país tiene características propias, como el silencio y la indiferencia de la sociedad en general, lo que tiende a empeorar por no hacer parte de las prioridades de la agenda pública. Recientemente, el gobierno saliente reorientó la política pública $^{1}$, ante las presiones que representa la sentencia T-025 de 2004 y autos posteriores $^{2}$, emanadas de la Corte Constitucional, que cataloga la situación en los siguientes términos: "El desplazamiento es un problema de humanidad que debe ser afrontado solidariamente por todas las personas, principiando, como es lógico, por los funcionarios del Estado (b) "un verdadero estado de emergencia social", "una tragedia nacional, que afecta los destinos de innumerables colombianos y que le marcará el futuro al país durante las próximas décadas" y "un serio peligro para la sociedad política colombiana."

No cabe duda que el tema del desplazamiento forzado en este contexto exige reflexiones urgentes, en ese sentido, este artículo resultado de la

${ }^{1}$ Es de resaltar un incremento sustancial de los recursos fiscales, la adopción de un Plan nacional de atención a la población desplazada, y un acompañamiento a los entes territoriales para el funcionamiento del sistema de atención integral a la población desplazada en todo el país.

La Corte constitucional hasta el año 2007, había emitido más de 40 fallos relacionados con la proble relática del Desplazam problemática del Desplazamiento
Forzado, incluyendo la sentencia T-025. 
${ }^{3}$ NARANJO GIRALDO, Gloria. (2001) "Reinvención de la identidad. Implicaciones del desplazamiento forzado en las culturas locales y nacional" En: Éxodo, Patrimonio e Identidad. V Cateara Anual de Historia Ernesto Restrepo Tirado, Bogotá, Museo Nacional. pp 78-103. Ver también García, M. (1999). Los desplazados por la violencia en Colombia. Con su dolor sin rumbo. En: Revista Universitas Humanística. S.J. 15 . investigación "Memoria, identidad, derechos y reconstrucción vital de la población desplazada en Cartagena", pretende realizar un aporte al tema, en lo relacionado con el proceso de reconstrucción vital de la población en situación de desplazamiento forzado, en el marco de la política pública establecida por el Estado colombiano. Para ello se ubica como radio de estudio el departamento de Bolívar, especialmente su distrito capital, Cartagena de Indias, ciudad declarada por la ONU, Patrimonio Histórico y cultural de la Humanidad en 1984, convertida en uno de los principales destinos turísticos del país y del Caribe.

En Cartagena el tema del desplazamiento forzado y sus consecuencias ha sido fuertemente invisiblizado por los intereses económicos, y la condición de ciudad con un entramado social que evidencia la secuela señorial y aristocrática de una sociedad colonial, la ausencia de un espíritu y carácter empresarial de riesgo para la actividad productiva, y una élite conservadora y costumbrista que carece de un proyecto transformador claro del entorno social, configurando lo que Anthony Gidenns(1993), denomina una sociedad de "modernidad tardía". Este modelo de sociedad excluyente, fragmentada y carente de un arreglo institucional que incluya este problema dentro de la agenda pública del distrito, explica porqué el desplazamiento forzado sigue ocupando un lugar marginal en los planes de desarrollo y las políticas públicas de los gobiernos departamental y distrital ${ }^{3}$.

Este escenario de indiferencia frente a un fenómeno de trascendencia nacional (en el país, la Corte Constitucional estima para el año 2009, 4 millones de desarraigados/ 800.000 familias en el territorio nacional) e internacional, como el desplazamiento forzado de miles de personas que lo perdieron todo y se encuentran en la ciudad de Cartagena, rehaciendo sus vidas y tratando de adaptarse y recuperar lo perdido obliga a estudiar el fenómeno detenidamente. De allí que esta investigación se propuso determinar cómo es la situación y ejercicio de los derechos económicos, sociales, políticos y culturales de la población desplazada en el distrito de Cartagena, contrastándolo con el rol de las políticas públicas y la responsabilidad institucional que ello significa, en el nivel local y regional.

La metodología utilizada en esta investigación se inscribe por un lado en una perspectiva de comprensión integral del problema y en el marco de la multidisciplinariedad dada la complejidad de variables que el fenómeno involucra en su génesis y desarrollo. Esa perspectiva de comprensión 
estructural y dinámica del problema pretende un esfuerzo académico por desentrañar el significado del desplazamiento dentro de la problemática global de la región y el país. En palabras de Mauricio García Duran:

“Una comprensión integral del problema es un reto para los investigadores, ya que es necesario un planteamiento que logre mostrar las relaciones que el fenómeno tiene con el contexto social y político del país y la manera como hace parte de dinámicas estructurales más amplias (procesos de exclusión) y la forma como es producto o generador de dichas dinámicas y conflictos. Es necesario poder comprender la dinámica e interacción de los distintos actores involucrados en el desplazamiento (tanto victimas como victimarios); es imprescindible poder entender cuáles son las dinámicas que en medio del dolor y la desarticulación social, pueden ofrecer caminos de salida y reconstrucción del proyecto de vida de los desplazados" (1999:17).

La visión multidisciplinar se concreta en la confluencia de varias disciplinas para el análisis del problema sin llegar a su tipificación y localización; sino que ante un problema de esta naturaleza y complejidad se exige creatividad, y diversas aproximaciones metodológicas para descubrir el quehacer y el cómo actuar frente a determinadas situaciones, coyunturas y circunstancias como las que muestra el desplazamiento forzado.

Complementa lo anterior el concepto de enfoque de Derechos el cual parte del reconocimiento de la persona como titular de derechos y como centro del proceso de desarrollo y de las políticas públicas, para garantizar el cumplimiento de los Derechos de los desplazados. A ello se agrega una revisión de los cuatro últimos Planes de Desarrollo Distrital y el Plan de Ordenamiento Territorial, el cual se encuentra aprobado desde el año 2001, con miras a verificar la definición de acciones y programas para la atención a las víctimas del desplazamiento forzado; y en igual sentido la articulación y correspondencia de las acciones de política pública entre el nivel nacional y el distrito.

\section{Situación de la población desplazada en Cartagena.}

Cartagena, es en la actualidad una ciudad que tiene una doble característica, de un lado experimenta un proceso de desarrollo sin antecedentes de su estructura urbana y sobre todo de la infraestructura portuaria y turística, lo que la convierte en una de las ciudades con mejores condiciones de competitividad portuaria en la actual globalización, y la posiciona como un destino turístico de primer orden en el Caribe. Y por otro lado de manera paralela con este

Palubra No. 11. Septiembre de 2009 - Agosto de 2010 
4 De acuerdo con el DANE para junio de 2005, del total de población ocupada322.069-, el 61:7\% es decir 198.298 trabajan en la informalidad y solo el $32 \%$ se ubica en la formalidad.

${ }^{5}$ Para el año 2005, el SISBEN nos señala que de un total de 629.478 sisbenizados 406.325 se ubican en el nivel $1 \mathrm{y} 192.040$ en el nivel 2 tan 192.040 en el nivel 2 tan solo 30.964 en el nivel 3 . En la medición anterio la cual cubre hasta el 2004 se encontraban 265.691 sisbenizados en el nivel 1; un total de 270.836 en el nive 2 y 104.773 en el nivel 3 , lo cual constata la afirmación sobre la "crisis social".

6 En el aspecto PolíticoInstitucional la ciudad es recurrentemente epicentro de escándalos sobre la depredación de los recursos públicos y la ausencia de gobernabilidad en la gestión pública.

${ }_{7}^{7}$ La misión para el diseño de una estrategia de reducción de la pobreza MERPD, estimó que la pobreza en Cartagena en el año 2005, afecta al $42 \%$ de la población. En igual sentido se calcula que

la población clasificada en nivel de indigencia es de un $10.3 \%$ del total de la población. desarrollo se encuentra signada por una grave crisis económica ${ }^{4}$, social ${ }^{5}$, política $^{6}$ e institucional, situación que se expresa en un empobrecimiento acelerado $^{7}$ de sus habitantes, lo que deriva hacia una creciente exclusión y marginalidad; e igualmente hacia una marcada debilidad de ciudadanía y de capacidad de defensa de lo público.

En otras palabras, la ciudad carece de un "Arreglo Institucional ", es decir de un conjunto de normas y/o reglas transparentes, estables que garanticen la interacción entre los diferentes actores ciudadanos y reduzcan la incertidumbre en el relacionamiento público-privado. En este apartado se precisa como la gestión pública distrital está soportada en una cultura arraigada de la informalidad y la improvisación, asociado a una discontinuidad en los planes de desarrollo y programas ejecutados, evidenciando que la ciudad no ha podido construir un "Sistema de Planeación Distrital", concebido como la instancia que armoniza y regula la actuación de los actores públicos y privados sobre el territorio distrital, en la perspectiva de construir una Cartagena ordenada, equitativa, sostenible y competitiva, que garantice la producción de bienes y servicios a la población localizada en su jurisdicción.

Esta realidad que afecta a las personas del común se evidencia de manera más dramática en la situación que enfrenta la población desplazada, ya que el Distrito se ha convertido en uno de los grandes centros de recepción de población víctima del desplazamiento forzado proveniente de todo el país; y en especial del departamento de Bolívar, en donde la dinámica del desplazamiento tiende a concentrarse en la región de los Montes de María, que ha tomado el papel de la zona expulsora-receptora del país con uno de los mayores números de población desplazada y en riesgo de desplazamiento. De acuerdo con las cifras del sistema de información de la población desplazada "SIPOD" de Acción Social, hasta a diciembre de 2009, en la ciudad de Cartagena residen 66.450 -equivalentes a 15.708 hogares- personas en condición de desplazamiento forzado.

Lo anterior es posible constatarlo al sopesar las cifras que arrojan la revisión de cien encuestas de familias víctimas del desplazamiento forzado registrada en Acción Social, las cuales fueron atendidas en la fase de Atención de Emergencia Humanitaria, por la entidad operadora "Corporación Minuto de Dios", durante los años 2005-2006.

Estas encuestas revelan aspectos preocupantes y estremecedores de esta 
"emergencia humanitaria". Quizá una de las mayores transformaciones está dándose en la estructura familiar de la población desplazada. La composición familiar muestra que la tipologia de familia "nuclear", es la de mayor predominio, con el $43 \%$ del total de las encuestas revisadas; seguido de la familia "extensa" con el 24\%; luego le siguen con el 14\% la familia "monoparental materna" y las personas que manifiestan vivir sin ninguna compañía. Estos cambios que se observan en la configuración familiar de los desplazados, tienen efectos sobre los roles y el tipo de relaciones al interior de la familia. Por ejemplo, al valorar la categoría de familia extensa (en esta conviven además de la familia nuclear "típica", los abuelos, tíos, primos, e incluso generaciones diferentes unidos por lazos de consanguinidad) y la familia monoparental materna (aquí solo tiene presencia la madre como cabeza del hogar), se evidencia el grado de complejización que van asumiendo las familias desplazadas en lo afectivo, el manejo de la autoridad, las pautas de crianza en un entorno citadino y muy distinto al de su lugar de residencia previo al desplazamiento.

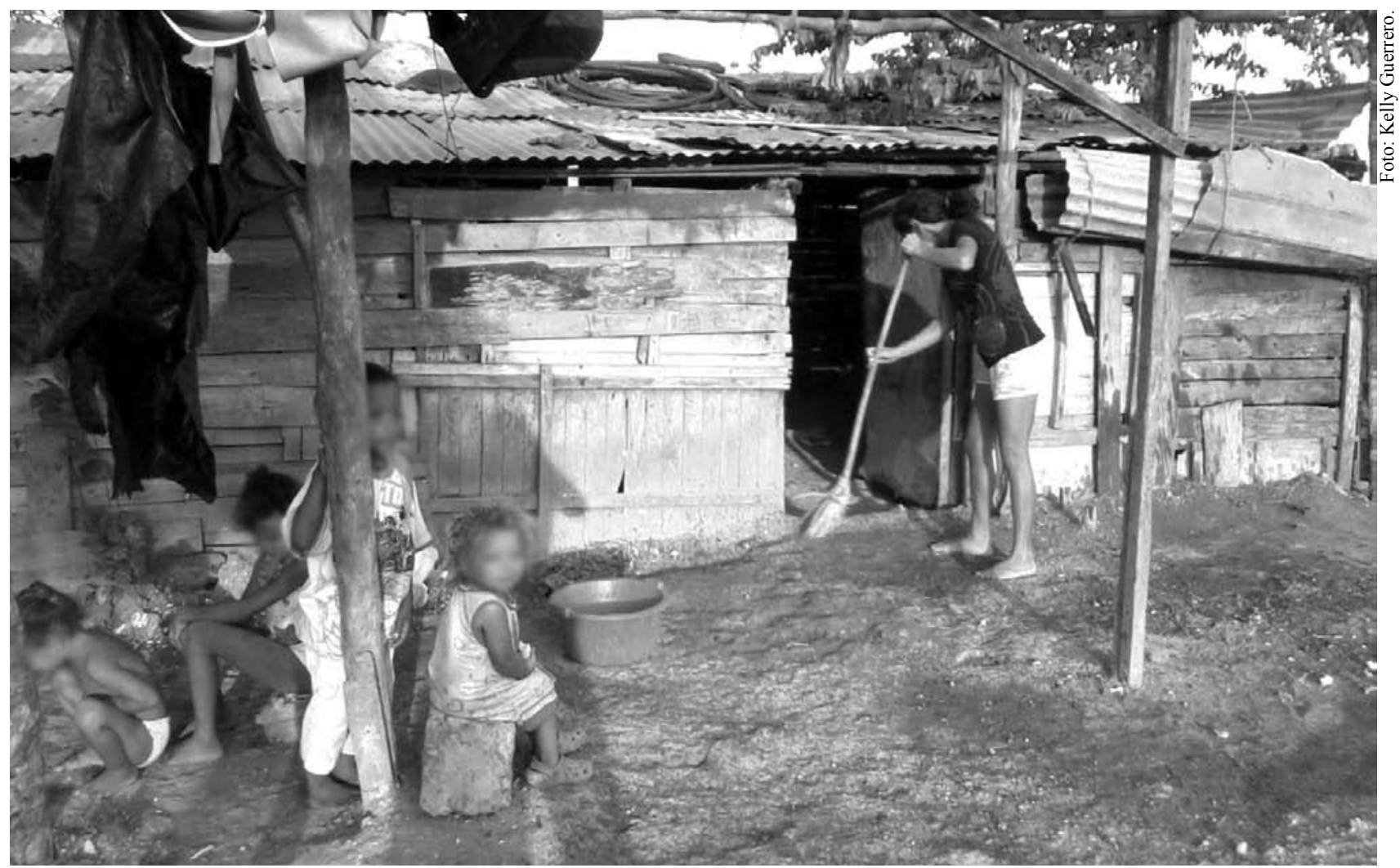

Esperando la visita. Cartagena 2011.

encuestas analizadas, manifiestan tener niños y niñas menores de 18 años. De allí se estiman en 231 niños y jóvenes menores de 18 años; se resalta como en la denominada "primera infancia", ${ }^{8}$ las familias reportan a 81 niños y 
8 La primera infancia incluye los niños desde la gestación hasta los cinco años sin haber cumplido los seis. Su importancia radica en que las iinvestigaciones efectuadas en varios campos del conocimiento - biología neurociencia, ciencias de comportamiento psicología del desarrollo, educacion y economía- concluyen y economía- concluyen que los primeros años de vida son básicos para el desarrollo del ser humano, en todos sus aspectos: biológico, psicológico, cultural y social. niñas, quienes ameritan un tratamiento acorde a su dinámica de crecimiento y desarrollo. De los restantes hogares, es decir en 21\%, los miembros superan los 18 años, por ejemplo entre los 19 y los 45 años se encuentran 141 personas, las cuales se ubican en plena etapa laboral. La composición por edad muestra hacia el futuro los costos (transitorios o permanentes) que en materia de educación, nutrición, salud y vivienda, debe asumir el Distrito de Cartagena, - a través de sus políticas públicas - con esta población.

En cuanto al lugar de expulsión las familias, en un 38 \% del total no informan de donde son oriundas, lo cual es posible atribuirlo a las condiciones de inseguridad y amenazas que afectan a esta población. El 26 \% de las familias reporta que son desplazadas del departamento de Bolívar; el 9\% provienen del departamento de Antioquia; el 7\% del Cesar; el 3\% de Córdoba y Sucre, el resto (9\%) son familias de lugares como el Chocó, Casanare, Magdalena, Nariño y de los departamentos de Santander del Sur y Norte de Santander. En relación a los municipios expulsores, para el caso del departamento de Bolívar, se precisan los municipios de El Carmen de Bolívar, Marialabaja, San Jacinto, Barranco de Loba y Tiquisio. El departamento de Antioquia, reporta los municipios de Turbo, San Francisco, San Pedro de Uraba, Dabeiba; El departamento del Cesar a La Jagua de Ibirico, Aguachica, Codazzi; Córdoba a Pueblo Nuevo; Sucre a Chalán, entre otros.

Al revisar lo relacionado con la ubicación de las familias desplazadas en el Distrito, se reconoce como los sitios de mayor concentración de esta población a los barrios de: El Pozón (19 familias); San José de los Campanos (12), Olaya Herrera (11), Nelson Mandela (7), Villa Hermosa (7), Henequén (5), el resto de familias se distribuye en 25 barrios o sectores de las localidades de la Virgen y de la Bahía. Es de señalar el cambio en la localización de la población desplazada en la ciudad, ya que en la década de los años 90 (cuando surge este proceso en el distrito), el lugar de mayor congregación de desplazados era el barrio Nelson Mandela; posteriormente en los últimos cinco años ha sido el Pozón, ahora el grueso de la población se dispersa en los diferentes sectores de las localidades ya reseñadas. Este cambio puede obedecer a factores de seguridad, o de ubicación cercanas a zonas de economía informal (por ejemplo el Mercado de Bazurto) para la generación de ingresos.

En cuanto al ejercicio de los derechos económicos, sociales y culturales, por parte de las familias encuestadas, se refleja el alto nivel de precariedad y vulnerabilidad en que se encuentran. Por ejemplo, en materia de afiliación 
al sistema de salud, el 80\% de los miembros de las familias no se hallan al momento de la encuesta como afiliados o beneficiarios del sistema; tan solo un $19.7 \%$ si goza del acceso a salud y seguridad social. Es llamativo como el $65 \%$ de los no afiliados manifiesta como motivos el desconocimiento de los procedimientos requeridos para ello. Es de resaltar que dentro de las Empresas Prestadoras de Salud, referenciadas están: SaludCoop, Mutual SER, Emdisalud, Coosalud y Solsalud entre otras.

En materia de activos económicos, el 56 \% de las familias reporta haber dejado tierras abandonadas en calidad de propietario antes del desplazamiento mientras que el $42 \%$ manifiesta no poseer tierras, y el $2 \%$ restante no informa al respecto. Similar situación acontece con la posesión de viviendas, ya que el $38 \%$ de las familias antes del desplazamiento era propietario y tan solo el $8 \%$ era arrendatario, el 35\% convivía con la familia y el resto (19\%) no informa al respecto. Por el contrario, la condición de desplazado, muestra para la fecha de la encuesta que el $47 \%$ se hallaba en el distrito en calidad de arrendatario, el 38\% en albergue donde familiares y amigos sin pagar arriendo y el restante $15 \%$ no informa al respecto.

En otras palabras, el contexto descrito permite afirmar, como efectos negativos del desplazamiento forzado en estos hogares asentados en la ciudad, los siguientes:

\) La pérdida de la tierra, de la vivienda y los activos de los hogares.

๑) La promoción del desempleo y la exclusión laboral

(7) El deterioro en las condiciones de salud, nutrición, una fragmentación del tejido social, $y$, en últimas contribuyendo al incremento sostenido de la pobreza en la ciudad.

$\square$ ) En igual sentido la vulnerabilidad y riesgo de los hogares desplazados en el mediano y largo plazo se incrementa ante la disminución ostensible del ingreso y consumo en los hogares, lo cual asociado a la fragilidad en la acumulación de nuevos activos e ingresos por parte de los programas estatales, facilita que los desplazados engrosen las filas de los pobres crónicos. (Ibáñez, 2008:168)

Estas afirmaciones las podemos corroborar revisando algunos apartes del estudio realizado por el Comité Internacional de la Cruz Roja9 en ocho ciudades de Colombia sobre la situación de la población desplazada, el cual incluye al Distrito de Cartagena y describe aspectos fundamentales para comprender

${ }^{9}$ Una mirada a la población desplazada en ocho ciudades de Colombia: Respuesta Institucional local, condiciones de vida y recomendaciones para su atención. CICRPMA. Bogotá. Diciembre de 2007.

Palubra No. 11. Septiembre de 2009 - Agosto de 2010 
mejor la tragedia que enfrentan las familias desplazadas que habitan la ciudad.

El proceso de inclusión de una persona como desplazado en el Registro Único de Población Desplazada RUPD, que maneja Acción Social, es en promedio de 23 días calendario desde el día de la declaración, hasta que recibe respuesta; de ocurrir retrasos el desplazado logra obtener su estatus como tal en 39 días. De acuerdo con la encuesta, las personas declaran en promedio 74 días calendario después de haberse desplazado.

En una segunda fase, luego de la incorporación al RUPD, la familia es remitida a la entidad operadora de Acción Social -actualmente es Children International (CHF)-, para ser beneficiaria en la atención de emergencia humanitaria durante al menos tres meses. En este proceso puede durar 8 días más, lo cual indica que en el mejor de los casos, una familia desplazada desde el momento de la declaración, hasta la recepción de la primera entrega de ayuda humanitaria transcurren 31 días calendarios.

La Unidad de Atención y Orientación al Desplazado " UAO”10, viene funcionado en el distrito desde el año 2004 -no obstante las limitantes de infraestructura, espacio y recurso humano-, se ha logrado avanzar en un protocolo de atención a la población desplazada. En ese esfuerzo concurren Acción Social, el Ministerio Público (Procuraduría, Defensoría y Personería) y el Distrito, a través de la Secretaría de Participación y Desarrollo Social. Las restricciones señaladas impiden al 60\% de las personas que acuden a declarar, alcanzar su cometido, ya que se quedan sin atención, ante las molestias y congestión del sitio. Es muy preocupante la ausencia en la UAO de las entidades con responsabilidad en las áreas de: salud, educación, vivienda, protección de tierras y seguridad alimentaria.

Las razones que argumentan los desplazados(as) para no declarar su condición -según la encuesta-, son entre otros: "no querer", miedo, el desconocimiento de los trámites o de los lugares donde debe realizarse. Otra razón de peso, la constituye la falta de credibilidad y confianza en el proceso. Esta percepción es resultante de la experiencia de otros desplazados que han pasado por el proceso y no han sido beneficiarios del mismo.

${ }^{10}$ Las oficinas de la UAO, funcionan en el barrio Getsemaní, en la antigua sede del CERCA. 
de un protocolo unificado y en general no es una atención sistemática, continua y en permanente monitoreo ante los cambios repentinos (ansiedad, estrés, tristeza, etc.) que experimenta la población víctima del desplazamiento forzado. Normalmente ante los problemas que afrontan los desplazados (ingresos, alimentación, vivienda, educación entre otros) en su cotidianidad, el tema de la salud mental pasa a un segundo plano. Durante los años 20052007, en la fase de atención de emergencia humanitaria se atendieron a 1.346 personas de 2.140 que llegaron en condición de desplazamiento a la ciudad; es decir solo se logró una cobertura del 60\%.

Este informe sin duda revela las complicaciones que toma el tema de los desplazados para ser sometido a las atenciones que demanda su condición; estas limitaciones del gobierno y de su precaria política pública para hacer frente a este fenómeno, hace que esta "tragedia nacional" tenga dificultades mayores y se convierta en un gran problema en la medida que los desplazados pierden la confianza en los procesos del Estado ya que este no brinda soluciones eficaces; visto de esta manera, las limitaciones institucionales serán cada vez mayores.

\section{Avances y limitaciones de la política pública para la atención de la población desplazada.}

Una de las debilidades de la política pública sobre desplazamiento forzado lo constituye el nivel de garantía y capacidad de respuesta por parte de las entidades estatales y territoriales que hacen parte del Sistema Nacional de Atención Integral al Desplazamiento Forzado SNAIPD, para hacer efectivo el goce de los derechos de la población afectada por este problema. Durante el proceso de investigación se logró visitar, intercambiar opiniones y revisar documentos ${ }^{11}$ con funcionarios y miembros de organizaciones de desplazados, que permiten afirmar entre otras:

Una limitante de carácter estructural para operativizar la política pública de atención a la población desplazada en el distrito lo constituye la carencia de un sistema de planeación territorial. En efecto, al revisar los cuatro últimos planes de desarrollo se constata la inexistencia de políticas, programas y proyectos dirigidos a la victimas del desplazamiento forzado. Los planes de Desarrollo Cartagena es Nuestra Casa (2004-2006) y Cartagena Siempre Nuestro Compromiso (2006-2007), Plan de Desarrollo Por Una Sola Cartagena (2008-2011) asignan recursos para la población desplazada en los programas destinados a mitigar la pobreza y la vulnerabilidad en la

${ }^{11}$ Por ejemplo se logró obtener y revisar el Plan Integral Único para la prevención y atención a la problemática del desplazamiento en el distrito de Cartagena. PIU.2007. 
12 Este tratamiento en la atención a los desplazados en el Distrito, es el resultado de la concepción que se genera en el sentido de que los desplazados con varios años de residir en la ciudad, ya no son desplazados, sino que hacen parte de los nuevos pobres de la ciudad.

${ }^{13}$ Es necesario reconocer que la administración distrital anterior (2006-2007) ante las presiones de las organizaciones de desplazadas y la sentencia de la Corte Constitucional decide ast mir su responsabilidad al respecto.

${ }^{14}$ El proceso de elaboración del PIU contó con el apoyo de Entidades como ACNUR, Acción social, Alcaldía Distrital, ONGS Organizaciones representantes de

la población desplazada

la población desplazada y miembros de universidades locales. ciudad $^{12}$. Es llamativo que en el Plan de Ordenamiento Territorial se "ignora "a los desplazados en su localización al interior de la ciudad y por ende no se establece programa alguno para esta población (por ejemplo en el tema de la vivienda), en el mediano y largo plazo. Lo anterior permite afirmar la incapacidad del distrito de Cartagena de formular una política pública para las víctimas del desplazamiento forzado; entendiendo la política pública como: las actuaciones de los gobiernos y otras agencias del Estado, cuando las competencias constitucionales así lo determinen, caracterizadas por que constituyen flujos de decisión-o una decisión específica-orientadas a resolver un problema que se ha constituido como público, que moviliza recursos institucionales y ciudadanos bajo una forma de representación de la sociedad que potencia o delimita esa decisión (2007:28).

Adicionalmente los niveles de coordinación, concurrencia y corresponsabilidad entre la nación (Acción Social y demás entidades del SNAIPD) y el distrito son excesivamente débiles y precarios, al momento de planear actividades, presupuestar recursos y posterior ejecución. Al contrastar en la gestión pública territorial como es la instrumentalización de las políticas públicas, se reflejan las severas limitaciones (ya son tradicionales) que tiene el Estado (Nacional/Territorial) en la formalización de las políticas públicas, tal como se evidencian a continuación:

】) El Comité distrital de atención a la población desplazada operaba como un ente informal y carente de direccionamiento estratégico hasta el año $2005^{13}$. A partir de ese momento y con el apoyo de ACNUR y demás entidades se logra dar continuidad y formalidad a la gestión del comité distrital. Desde el año 2006, se ha regularizado la operación del comité, además se destaca la conformación de mesas de trabajo en las áreas de salud, educación, vivienda y seguridad alimentaria esencialmente.

—) A partir de ese esfuerzo se culmina la elaboración del Plan Integral Único de Atención a la Población Desplazada ${ }^{14}$, durante los años 2006 y 2007. Es de resaltar que el PIU establece como líneas prioritarias: la prevención y protección de la población desplazada, la atención humanitaria de emergencia, el acceso a educación, salud, vivienda; la generación de ingresos y la participación de los hogares. Se establece una inversión durante los próximos cuatro años de \$158.820.640.640, entre las diferentes instancias responsables de la política pública (la nación, debe aportar el $75 \%$ y el distrito y el departamento de Bolívar el restante 25\%). 
Para el primer año, se estima invertir en la población desplazada residente en el distrito la suma de \$32.479.956.

๑) Dentro de las metas que el PIU plantea lograr durante el periodo 20082011, se ponderan en materia de aseguramiento en salud cobijar a 36.000 personas (actualmente son 12.000); la construcción de 2.421 viviendas, es decir darle respuesta a un $22 \%$ de la demanda existente. Al respecto es válido preguntar dada la recurrente fragilidad de las finanzas públicas territoriales y el tradicional incumplimiento del Estado ies viable alcanzar estas metas de inversión?

\) A partir del año 2006, se decide por parte de Acción Social la entrega de subsidios condicionados a la población desplazada a través del programa Familias en Acción ${ }^{15}$. En el caso de Cartagena, este proceso inicia en el año 2007 y a la fecha un total de 5.779 niños y niñas reciben subsidios educativos y 3.023 beneficiarios se les suministran recursos para nutrición.

\) En cuanto al ejercicio del derecho a la educación es de reseñar como para el año 2006, por primera vez la Secretaria de Educación Distrital maneja un registro sobre la población desplazada que logra acceder al sistema escolar. Para ese año aparecen matriculados un total de 4.004 estudiantes que acreditan la condición de desplazado, de los cuales 2.138 pertenecen al género masculino y 1.886, al femenino. De ese total de estudiantes 1.909 cursaron la básica primaria y 1.424 la secundaria y en la fase de transición se ubican a 309 estudiantes. Los barrios en donde se localizan los establecimientos educativos son entre otros: El Pozón, Nelson Mandela, Olaya Herrera, Arroz Barato, San José de los Campanos, Pasacaballos, Escallón Villa, Blas de Lezo y María Cano. Para el año 2007, de acuerdo con cifras del PIU, se reporta una matrícula de 4.726 estudiantes en situación de desplazamiento; al confrontar esta cobertura con el total de población en edad escolar que registra el SIPOD para la fecha de 15.163 niños y jóvenes, equivale solo al 31\% de la población, en otras palabras 10.887 estudiantes están por fuera del sistema educativo distrital. Es importante destacar como los niños y jóvenes que asisten a los establecimientos educativos están exentos de los costos de pensión y matrícula, adicionalmente en el año 2006 el ICBF suministró 1.027 kits escolares y 5.500 raciones alimenticias.

Estos esfuerzos en materia educativa siguen siendo insuficientes para

${ }^{15}$ El programa Familias en Acción entrega a sus beneficiarios recursos monetarios para la nutrición y educación de los niños y niñas que asisten a la escuela cada dos meses. 
reducir los índices en materia de analfabetismo, el PIU señala que de acuerdo con una encuesta efectuada en la población desplazada en el distrito, se estima que un 30\% -2.546 personas- manifiestan "no saber leer ni escribir". De ese total el $47 \%$ son mujeres y el $53 \%$ son hombres.

7) El Departamento Administrativo Distrital de Salud DADIS, precisa que hasta el año 2007, el total de personas desplazadas afiliada al régimen subsidiado ascendía a 12.647, equivalentes al 26\% del total registrado por el SIPOD durante este año (47.910). Es de resaltar que ese año se asigna un cupo de 6.000 beneficiarios, el cual no puede ser completo ante la no presencia de las personas incorporadas en el SIPOD y en el SISBEN. Como factores restrictivos que los desplazados usuarios del régimen padecen se reconocen 1) La precariedad en la atención por parte de las EPS/IPS. 2) La deficiencia en el suministro de medicamentos. 3) La oportunidad y eficiencia en la prestación del servicio. El 69 \% de la encuesta realizada a la población desplazada por la Organización Panamericana de la Salud manifiesta su insatisfacción con los servicios prestados. Es pertinente señalar que el DADIS suministra en el año 2006, un total de 12.273 dosis de vacuna contra el polio; 744 dosis para la DPT; 637 dosis para la triple viral y 669 antimalárica.

๑) En cuanto a la nutrición y seguridad alimentaria desde el año 2007, un total de 3.708 familias vienen recibiendo los subsidios de nutrición que el Programa Familias en Acción le entrega a sus beneficiarios. Asimismo el Instituto Colombiano de Bienestar Familia ICBF, durante los años 20052006, logra cobijar a 13.197 niños y niñas en su programa de alimentación y mejoramiento nutricional. En ese mismo periodo vincula a 772 víctimas del desplazamiento al proyecto de Madres Gestantes. Por su parte el DADIS reporta haber vinculado a 959 niños y niñas y 357 madres lactantes en su programa de la vigencia 2006.

๑) En cuanto al ejercicio del derecho a la vivienda tan solo se ha logrado la asignación de 823 subsidios de vivienda por parte del Ministerio del Medio Ambiente. en el año 2004. De ese total tan solo 94 beneficiarios acceden a su vivienda, los restantes 665 están a la espera de la ejecución de los proyectos. Este es uno de los derechos más vulnerados en la población desplazada residente en Cartagena y que refleja la precariedad y limitada capacidad de acción de las entidades responsables, en este caso CORVIVIENDA. 
—) El desarrollo organizacional de la población desplazada se evidencia en la activa presencia de organizaciones sociales como: la coordinación nacional de desplazados CND, la Mesa Nacional Indígena; Andescol, Convergencia y Afrodes. Las organizaciones reseñadas hacen parte de instancias como el Comité Distrital de Atención a la Población Desplazada y sus diferentes grupos de trabajo, como la mesa educativa, la mesa de salud etc. Es de particular interés describir las condiciones de seguridad de los dirigentes y lideres de las organizaciones sociales de desplazados, en la ciudad. En efecto dada la creciente inseguridad en los barrios y zonas en donde se localizan este segmento de la población ${ }^{16}$, la Defensoria del Pueblo elabora un análisis de riesgo, en donde 58 personas representantes de organizaciones de desplazados, deben otorgárseles medidas de protección. A la fecha a 33 de estas personas el Ministerio del Interior les ha asignado algunas acciones de protección y el Distrito de Cartagena a 22 personas con niveles de riesgo.

—) La actual administración en su plan de desarrollo Por una Sola Cartagena, concentra todos sus esfuerzos y acciones para combatir la pobreza y la exclusión social (artículo 4 del plan), en el Plan de Emergencia Social Pedro Romero PES, articulando los programas y recursos nacionales (Red Juntos, Familias en Acción y Atención a Población Desplazadas), con la acción distrital. No obstante las actividades emprendidas (al momento de culminar esta investigación), el PES aún se encuentra en la etapa de despegue su operación, si bien se reconoce su importancia como herramienta para combatir la pobreza extrema y la exclusión social; es indispensable que el PES cuente con un sistema de seguimiento y evaluación que permita establecer con certeza sus resultados e impacto durante las distintas fases del programa.

Pese al direccionamiento de la política pública y algunos logros para atender la tragedia social que presenta la población desplazada, aún sigue siendo insuficiente y discontinuo este esfuerzo, razón por la cual las víctimas del desplazamiento siguen siendo incrédulas frente a una política de Estado que no solo es incompleta y limitada, sino que además genera incertidumbre y desconfianza. De esta manera la amenaza que representa el desplazamiento para la integración social del país se convierte como lo señaló la Corte Suprema de Justicia en "un serio peligro para la sociedad política colombiana".

${ }^{16}$ Específicamente barrios como El Pozón, Nelson Mandela, Olaya Herrera, San José de los Campanos etc. se han venido presentando en los últimos cinco ños operativos de "Limpiea Social", y han sido asesinados reconocidos líderes de nados reconocidos líderes
la población desplazada. 


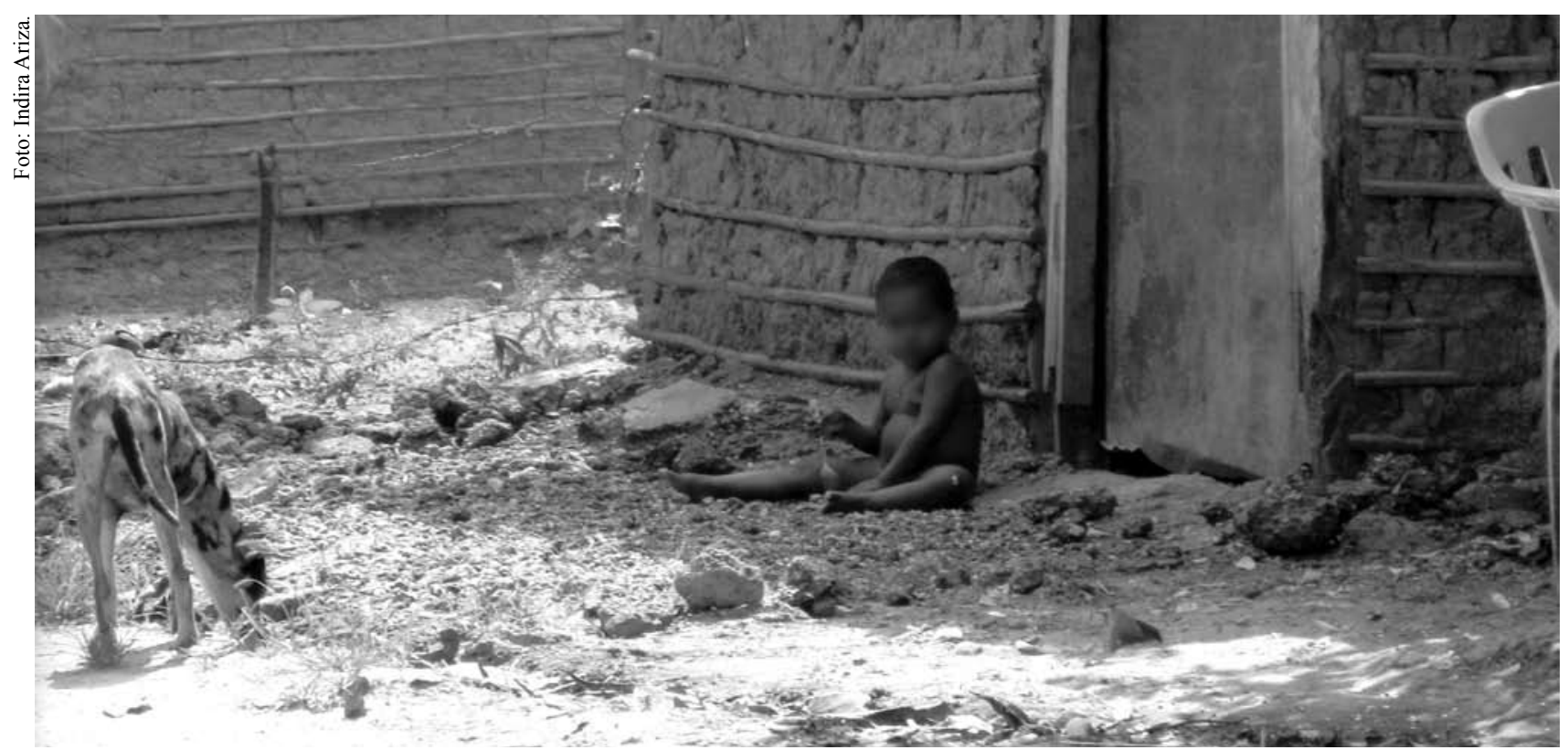

Jugando en el patio. Cartagena 2011.

\section{Conclusiones y recomendaciones.}

Transcurridos más de diez años desde la expedición de la ley 387 de 1997, de sus decretos reglamentarios y la reciente sentencia T-025 de la Corte Constitucional, es innegable que la atención a la población desplazada en el Distrito muestra tímidos avances en relación a la magnitud del problema y los desarrollos en otras ciudades y zonas del territorio nacional. Estos avances significan la continuidad de los procesos de atención de emergencia, algunos progresos en la institucionalización del Comité Distrital, y la formulación del Plan Integral Único de Atención a la Población Desplazada, sin embargo como limitante transversal se reconoce que prevalece la "mentalidad de la ayuda y no la de ejercicio de los derechos económicos, sociales y culturales". Esta actitud es el resultado de la construcción de una sociedad en la que pesan o subsisten imaginarios de segregación social y discriminación racial (típicos de sociedades de modernidad "tardía"), contrario a una cultura de los Derechos Humanos como elemento fundante y soporte de una sociedad moderna y democrática.

Un reto ineludible e inaplazable para el distrito de Cartagena en un contexto de la descentralización de las políticas públicas, lo constituye el de lograr alcanzar una concertación en la adopción y ejecución de la política pública de desplazamiento forzado, entre el nivel nacional (Acción Social-Ministerios y demás entes), el nivel departamental y la administración local. Esta concertación debe partir de unas reglas de juego desde la asignación de 
recursos, la definición clara de las competencias y responsabilidades de cada ente territorial, de tal manera que el conjunto agregado de las políticas garantice el acceso efectivo a los derechos ciudadanos de los desplazados. (Pedraza y Restrepo, 2007:95). A ello se adiciona la inaplazable necesidad de construir un Sistema de Planeación Distrital como soporte de la formulación y ejecución de políticas públicas territoriales que posibiliten el goce efectivos de los derechos a la población desplazada.

En otras palabras, la corresponsabilidad del Distrito en la atención a las víctimas del desplazamiento forzado puede entenderse en función de la capacidad fiscal e institucional como tal, y de las condiciones del desplazamiento en la ciudad. Luego entonces la política pública debe ajustarse sobre la base de reconocer las diferencias en la capacidad de gestión institucional. Lo anterior permite entender la ausencia de una adecuada coordinación interinstitucional, al momento de formular el PIU Distrital. Por ejemplo, el PIU en su elaboración tiene como fundamento la realidad local, pero la mayor parte de los recursos presupuestales se definen en el nivel nacional, lo cual se convierte en una limitante ante factores como: A) La inversión de la nación tiene un énfasis sectorial (vía ministerios y Acción Social), lo cual muchas veces no se logra concretar con las metas distritales, como en el caso de la atención psicosocial o el impulso a la consolidación de las organizaciones de desplazados y los mismos proceso de retorno / restablecimiento de la población desplazada (B). En igual sentido, la asignación de recursos está fuertemente centralizada y concentrada, en tres instituciones (Acción Social, Ministerio de la Protección Social y Ministerio de Educación Nacional), quienes cobijan un 80\% de los recursos apropiados para atender a la población desplazada, durante los años 2005-2007 (ACNUR, 2007).

Adicional a lo anteriormente descrito, es urgente la creación de esa instancia de concertación, para que el distrito pueda participar en la definición de las políticas públicas de atención a las víctimas del desplazamiento forzado, a fin de que finalmente el distrito deje ese rol de simple "instrumentalizador" en la realidad de la política pública. 


\section{BIBLIOGRAFIA}

Alcaldía Mayor de Cartagena. (2001) Plan de Ordenamiento Territorial del Distrito Turístico y Cultural de Cartagena de Indias. Decreto No 0977. Cartagena: Secretaria de Planeación distrital.

. (2005). "Cartagena es nuestra Casa". Plan de Desarrollo (20042006). Cartagena: Secretaria de Planeación distrital.

- (2006). "Cartagena Siempre Nuestro Compromiso". Plan de Desarrollo (2006-2007) Cartagena: Secretaria de Planeación distrital.

- (2008). "Por una Sola Cartagena". Plan de Desarrollo (2008-

2011).Cartagena: Secretaria de Planeación distrital.

- (2007). Plan Integral único para la prevención y atención a la problemática del desplazamiento en el distrito de Cartagena -PIU- Cartagena.

Agencia Presidencial para la Acción Social y la Cooperación Internacional (2007). Programa Familias en Acción. Bogotá: Presidencia de la República.

CICR-PMA. (2007). Una mirada a la población desplazada en ocho ciudades de Colombia: Respuesta Institucional local, condiciones de vida y recomendaciones para su atención. Bogotá.

Corporacion Minuto de Dios. (2005). Encuestas a hogares población desplazada, Cartagena.

CUERVO, Jorge (2007). Las políticas públicas: Entre los modelos teóricos y la práctica gubernamental (una revisión a los presupuestos teóricos de las políticas públicas en función de su aplicación a la gestión pública colombiana). En: Ensayos sobre Políticas Públicas. Bogotá: Universidad Externado de Colombia.

DANE (2005). Departamento Administrativo Nacional de Estadística. CENSO NACIONAL DE POBLACION. Bogotá.

IBÁÑEZ, Ana (2008). El desplazamiento Forzoso en Colombia: Un Camino sin Retorno hacia la Pobreza. Bogotá: Universidad de los Andes. CEDE. Ediciones Uniandes 2008.

GARCIA, Mauricio (1999). Los desplazados por la violencia en Colombia. Con su dolor sin rumbo. En Revista Universitas Humanística. S.J. (15). Bogotá: Universidad Javeriana.

GIDDENS, Anthony. (1993). Consecuencias de la Modernidad. Madrid: Alianza Editorial.

Misión para el diseño de una Estrategia para la Reducción de la Pobreza y la Desigualdad (MERPD). (2006). Metodología de medición y magnitud de la pobreza en Colombia, Bogotá.

NARANJO, Gloria (2001). “Reinvención de la identidad. Implicaciones del desplazamiento forzado en las culturas locales y nacional" En: Éxodo, Patrimonio e Identidad. V Cátedra Anual de Historia Ernesto Restrepo Tirado. Bogotá: Museo Nacional.

PEDROZA, Betty y RESTREPO, Darío. (2007). Las entidades territoriales en la realización de derechos de población desplazada. Bogotá.

Corte Constitucional de Colombia. (2004). Sentencia T-025. Bogotá. 